$1-20-2005$

\title{
Fractionation of Cell Mixtures Using Acoustic and Laminar Flow Fields
}

\author{
Manoj Kumar \\ Cleveland State University \\ Donald L. Feke \\ Case Western Reserve University \\ Joanne M. Belovich \\ Cleveland State University
}

Follow this and additional works at: https://engagedscholarship.csuohio.edu/encbe_facpub

Part of the Biochemical and Biomolecular Engineering Commons

How does access to this work benefit you? Let us know!

\section{Publisher's Statement}

This is the accepted version of the following article: Kumar, M., Feke, D. L., , \& Belovich, J. M. (2005). Fractionation of cell mixtures using acoustic and laminar flow fields. Biotechnology and Bioengineering, 89(2), 129 - 137. doi:10.1002/bit.20294, which has been published in final form at http://onlinelibrary.wiley.com/doi/10.1002/bit.20294/abstract

\section{Original Citation}

Kumar, M., Feke, D. L., , \& Belovich, J. M. (2005). Fractionation of cell mixtures using acoustic and laminar flow fields. Biotechnology and Bioengineering, 89(2), 129 - 137. doi:10.1002/bit.20294

\section{Repository Citation}

Kumar, Manoj; Feke, Donald L.; and Belovich, Joanne M., "Fractionation of Cell Mixtures Using Acoustic and Laminar Flow Fields" (2005). Chemical \& Biomedical Engineering Faculty Publications. 38.

https://engagedscholarship.csuohio.edu/encbe_facpub/38

This Article is brought to you for free and open access by the Chemical \& Biomedical Engineering Department at EngagedScholarship@CSU. It has been accepted for inclusion in Chemical \& Biomedical Engineering Faculty Publications by an authorized administrator of EngagedScholarship@CSU. For more information, please contact library.es@csuohio.edu. 


\title{
Fractionation of Cell Mixtures Using Acoustic and Laminar Flow Fields
}

\author{
Manoj Kumar, ${ }^{1 *}$ Donald L. Feke, ${ }^{2}$ Joanne M. Belovich ${ }^{1}$ \\ ${ }^{1}$ Department of Chemical and Biomedical Engineering, \\ Cleveland State University, Cleveland $\mathrm{OH}$ 44115-2425 \\ ${ }^{2}$ Department of Chemical Engineering, Case Western Reserve University, \\ Cleveland, $\mathrm{OH} 44106-7217$
}

\begin{abstract}
A fractionation method applicable to different populations of cells in a suspension is reported. The separation was accomplished by subjecting the suspension to a resonant ultrasonic field and a laminar flow field propagating in orthogonal directions within a thin, rectangular chamber. Steady, laminar flow transports the cell suspension along the chamber, while the ultrasonic field causes the suspended cells to migrate to the mid-plane of the chamber at rates related to their size and physical properties. A thin flow splitter positioned near the outlet divides the effluent cell suspension into two product streams, thereby allowing cells that respond faster to the acoustic field to be separated from those cells that respond more slowly. Modeling of the trajectories of individual cells through the chamber shows that by altering the strength of the flow relative to that of the acoustic field, the desired fractionation can be controlled. Proof-of-concept experiments were performed using hybridoma cells and Lactobacillus rhamnosus cells. The two populations of cells could be effectively separated using this technique, resulting in hybridoma/Lactobacillus ratios in the left and right product streams, normalized to the feed ratio, of $6.9 \pm 1.8$ and $0.39 \pm 0.01$ ( $\mathrm{vol} / \mathrm{vol})$, respectively. The acoustic method is fast, efficient, and could be operated continuously with a high degree of selectivity and yield and with low power consumption.
\end{abstract}

Keywords: cell separations; acoustics; bioseparations; field-flow fractionation

\section{INTRODUCTION}

Homogeneous populations of cells are needed for numerous applications in biomedicine and biotechnology, such as in the separation of white blood cells from red blood cells and platelets, separation of pancreatic cells from adipose and acinar tissue, and purification of stem cells from bone marrow samples. Numerous approaches have been adopted in the past to concentrate specific cell populations. Separation of cells by sedimentation using inclined settlers (Davis et al., 1991) has been shown to be effective in separating viable from nonviable hybridoma cells. Density centrifugation has been used for fractionation of cell populations, but this method is difficult to scale for handling large flow rates. Fluorescence activated cell sorting (FACS) has been scaled-up but the equipment is expensive and requires labeling of cells (Simon et al., 1998). Affinity-based methods, such as immuno-adsorption and magnetic methods (Hartig et al., 1996; Svoboda, 2000; Ximena et al., 1997), are scaleable but also require antibody labeling.

In 1988, Giddings introduced a split-flow fractionation process (SPLITT) that separated different sized particles in a thin, rectangular chamber by using gravitational and flow fields aligned perpendicular to each other. This method was used to fractionate populations of micron-scale solids as well as cells (Giddings, 1992; Giddings et al., 1991). In the SPLITT method, processing rates are limited by the response of particles to gravity that, owing to their small size, is often very slow.

In the present work, we enhance this separation concept for cell populations by applying a resonant acoustic field normal to the flow direction. Acoustic forces on suspended cells can greatly exceed gravitational forces, and hence efficient separation of cell populations may be anticipated. The acoustic technique separates the cells based on differences in size, density, or compressibility. The method provides a fast, continuous, low-power consumption, and high purity processing method that can be scaled up.

The fractionation of cells is performed in a rectangular channel having a gap that is narrow relative to its lateral dimensions. Figure 1 provides a schematic of the separation concept. Shown is a chamber consisting of a planar acoustic transducer and a parallel reflector set one-half acoustic wavelength apart. The feed suspension enters the bottom of the chamber near the transducer wall. An inlet splitter restricts the lateral position of the feed, and a carrier fluid is introduced to the left of the inlet splitter in a manner that maximizes straight fluid streamlines throughout the chamber. Near the exit of the chamber, an outlet splitter is positioned just to the right of the chamber mid-plane. 


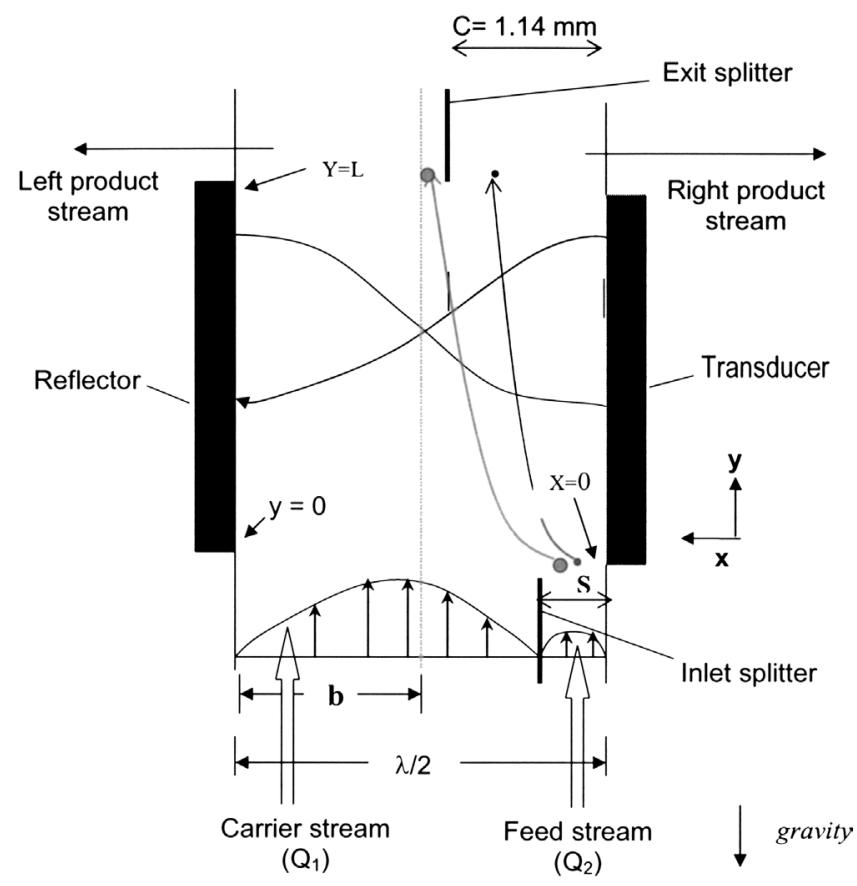

Figure 1. Representation of the acoustic fractionation concept.

When the transducer is energized at the appropriate resonant ultrasonic frequency, an acoustic pressure node is formed at the chamber mid-plane and pressure antinodes are formed at the chamber walls (Mandralis et al., 1990). Provided that the acoustic wavelength is large compared to the size of the suspended particle, the primary acoustic force $F_{a c}$ acting on an individual suspended particle is given by (King, 1934; Yosioka and Kawasima, 1955):

$$
F_{a c}=4 \pi R^{3} \kappa E_{a c} F \sin (2 \kappa x)
$$

where $\kappa$ is the wave number of the applied sound, $E_{a c}$ is the average acoustic energy per unit volume, $R$ is the sphere radius, $x$ is the position in the fluid relative to an acoustic pressure antinode, and $F$ is the acoustic contrast factor. $F$ depends on the densities of the suspending fluid and the solid, and the relative compressibility of the fluid and solid, given by (Mandralis and Feke, 1993):

$$
F=\frac{1}{3}\left[\frac{5 \rho_{p}-2 \rho_{f}}{\rho_{f}+2 \rho_{p}}-\frac{\gamma_{p}}{\gamma_{f}}\right]
$$

Here, $\rho_{\mathrm{p}}$ is the density of the particle, $\rho_{\mathrm{f}}$ is the density of carrier fluid, $\gamma_{p}$ is the compressibility of particle, and $\gamma_{f}$ is the compressibility of carrier fluid. Previous work has shown that particles with positive acoustic contrast factor move towards the pressure nodes and, conversely, particles with negative $F$ move towards the pressure antinodes in the absence of any other forces acting on them (Hawkes et al., 1998a, b; Johnson and Feke, 1995; Mandralis and Feke, 1993).
Based on these concepts, Mandralis and Feke (1993) demonstrated the size-fractionation of a population of 2$30 \mu \mathrm{m}$ polystyrene particles in aqueous suspension. Since no inlet splitter was used in this work, fractionation efficiency was relatively low. Incorporation of an inlet flow splitter and carrier stream by Johnson and Feke (1995) significantly improved separation efficiency. In the current work, the method is further improved by incorporating the design features for the acoustic chamber suggested by Rusinko (2000) to optimize the acoustic field quality. This design approach has been successfully demonstrated in studies of the acoustically driven motion of particles (Grossner et al., 2004) and in the coalescence of emulsified droplets (Pangu and Feke, 2004). Here, for the first time we attempt to integrate these various methods and demonstrate the fractionation of a binary mixture of cell populations. Fractionation of cell suspension was expected to present a great challenge for a number of reasons. These include the fact that cells have a susceptibility to acoustic forces that is somewhat smaller than that of polystyrene particles used to demonstrate other acoustic separation processes. In addition, there is a relatively wide spread in the size distribution, the cells may not be spherical, and may tend to form clusters.

Since the cell density is higher and its compressibility is lower than that of the suspension media, each type of cell is expected to have a positive acoustic contrast factor. The acoustic force on the cells acting laterally within the chamber scales as $R^{3} F$. The hydrodynamic drag on the cells resisting this motion scales with the cell radius $R$ according to the Stokes drag law. Thus, the net lateral speed of motion of the cells depends on $R^{2} F$. Since the variation of $F$ between the cell types is expected to be relatively small, we expect larger cells will be driven to the node faster than smaller cells. Individual cell trajectories can be manipulated by controlling the relative strengths of the laminar flow field and resonant acoustic field.

\section{MODEL FOR THE SEPARATION PROCESS}

A process model based on analysis of cell trajectories through the chamber can be used to assess the potential sharpness of the fractionation method and to benchmark the experimental results. Following Mandralis et al. (1990), the balance of acoustic and drag forces on the cell in the $x$-direction is:

$$
-6 \pi \mu R \frac{d x}{d t}+4 \pi R^{3} \kappa E_{a c} F \sin (2 \kappa x)=0
$$

where $\mu$ is the fluid viscosity and $x$ is the distance from a pressure antinode. An inherent assumption in Giddings (1988) is that there is no fluid motion in the $x$-direction. Similarly, the balance of gravitational and drag forces acting on the cell in the $y$-direction is given by:

$$
6 \pi \mu R\left(v_{y}-\frac{d y}{d t}\right)-m g=0
$$


with:

$$
m=\frac{4}{3} \pi R^{3}\left(\rho_{p}-\rho_{f}\right)
$$

where $v_{y}$ is the vertical component of the bulk fluid velocity imposed in the chamber, $m$ is the mass of the particle, $\rho_{p}, \rho_{f}$ are the densities of the cell and the carrier fluid, respectively. Here we also assume the cell to be spherical for the purposes of computing the gravitational and buoyant forces.

Equation 3 is made dimensionless and integrated analytically to determine the $x$-component of the trajectory of a cell having initial lateral location $x_{\mathrm{o}}$ :

$$
x^{*}=\frac{2}{\pi} \arctan \left[\tan \left(\frac{\pi x_{o}^{*}}{2}\right) \exp \left(\pi D t^{*}\right)\right]
$$

Here $x^{*}=x / b$, with $b$ the distance between the transducer and chamber mid-plane, and $t^{*}=t Q / 2 b L$, where $L$ is the transducer length, $Q$ is the fluid volumetric flow rate per unit depth of the chamber, and:

$$
D=\frac{4}{3} \frac{R^{2} E_{a c} L \kappa F}{\mu Q}
$$

The dimensionless parameter $D$ can be interpreted as the ratio of the acoustic force on the cell relative to the viscous drag it experiences due to its transverse motion across the chamber.

In dimensionless form, Eq. 4 becomes:

$$
\frac{d y^{*}}{d t^{*}}=\frac{3}{2} x^{*}\left(2-x^{*}\right)-J
$$

where $y^{*}$ is defined as $y / L$, the first term on the right represents the laminar flow profile within the chamber (assumed to be fully developed laminar flow), and:

$$
J=\frac{4}{9} \frac{R^{2} b\left(\rho_{p}-\rho_{f}\right) g}{\mu Q}
$$

The dimensionless quantity $J$ represents the ratio of the net gravitational force on a cell to the viscous drag force it experiences in the vertical direction.

Given that the physical properties of the cells and the geometric and operating parameters of the chamber are known, Eqs. 6 and 8 can be solved numerically using the Runge-Kutta method to obtain the individual trajectories for cells. A range of starting positions between the transducer and inlet splitter can be assumed and the $x$ position at the chamber exit $(y=L)$ can be calculated. Assuming a uniform distribution of entrance positions, the fraction of cells that exit the chamber in each of the two product streams can be computed.

One parameter that is necessary for the trajectory calculations but is not possible to measure directly is the acoustic energy density (the sum of kinetic and potential energy associated with the sound field) within the chamber. Typically, acoustic energy density is inferred from measurements of other process variables. For example, hydrophones can be used to measure acoustic pressure within a chamber, which is then correlated with acoustic energy density through the appropriate physical models. However, in our case, with relatively small chamber dimensions, even miniature hydrophones could interfere with the propagation of the acoustic field. Instead, we measure the voltage applied to the driving transducer and use the multilayer resonator model of Rusinko (2000) to compute the acoustic energy density within the chamber. This model has proved successful in other applications (Mandralis and Feke, 1993; Pangu and Feke, 2004).

The acoustic contrast factor of the hybridoma cells was determined using an acoustic levitation technique. In this method, an acoustic chamber is oriented such that the direction of propagation of the acoustic field is aligned with gravity. A reference particle with known acoustic properties (e.g., polystyrene) is introduced to the chamber, and the minimum transducer voltage $V_{\text {min,ref }}$ required to levitate the particles is measured. Similarly, the minimum transducer voltage required to levitate the cell $V_{\text {min,cell }}$ is measured. The scaling relationship:

$$
F_{\text {cell }}=\frac{\left(\rho_{\text {cell }}-\rho_{\text {fluid }}\right)\left(V_{\text {min }, \text { ref }}\right)^{2}}{\left(\rho_{\text {ref }}-\rho_{\text {fluid }}\right)\left(V_{\text {min }, \text { cell }}\right)^{2}} F_{\text {ref }}
$$

connects the acoustic contrast factor of the cells $\left(F_{\text {cell }}\right)$ to that of the polystyrene, $F_{\text {ref. }}$. This method resulted in $F_{\text {cell }}=$ 0.16 for hybridomas. $F_{\text {cell }}$ was calculated to be 0.11 when using physical properties of red blood cells $\left(\rho=1.093 \mathrm{~g} / \mathrm{cm}^{3}\right.$, $\gamma=3.38 \times 10^{-10} \mathrm{~m}^{2} / \mathrm{N}$ [Toubal et al., 1999]). The density of bacteria ranges from $1.031-1.212 \mathrm{~g} / \mathrm{cm}^{3}$ (Sharma et al., 1998). Assuming that the speed of sound (c) in bacteria is similar to that in red blood cells, the bacteria compressibility can be calculated from the relationship $\gamma=$ $1 /\left(\rho c^{2}\right)$. From these values, the acoustic contrast factor for bacteria is calculated to be $0.10-0.19$ (mean $=0.15$ ). In the simulations in this work, $F_{\text {cell }}=0.16$ was used for hybridomas and $F_{\text {cell }}=0.15$ used for lactobacilli.

\section{Acoustic Chamber Design}

The chamber is constructed of polyacrylamide and consists of three main components: the centerpiece which holds the inlet and outlet splitters as well as the Teflon spacers, and the two support structures that house the transducer and reflector, and which have openings for the inlet and outlet fittings (Fig. 2). The inlets and outlets are designed with a right-angle bend in tubing to reduce the turbulence in the chamber. The Teflon spacers are designed to be at an angle to the splitters to promote smooth flow of the fluid into the chamber.

The transducer and reflector are affixed to two adjustable positioning plates each by using double-sided adhe- 


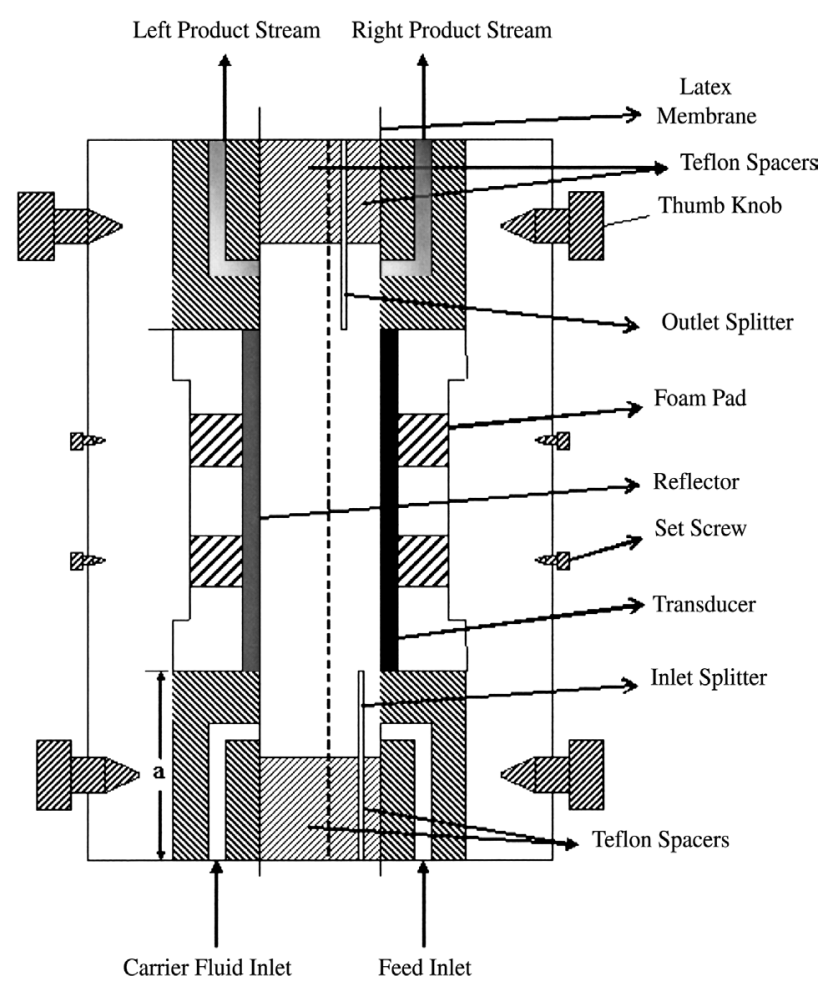

Figure 2. Schematic of the cross-section of the acoustic-flow fractionation chamber.

sive foam pads. Rigid attachment of the transducer to the supporting structure normally leads to vibration of the transducer in transverse modes; besides, its characteristic vibration is in the thickness mode. These vibrations have been avoided by attaching the transducer and reflector to the frame via a latex membrane $(0.035 \mathrm{~cm}$ thick). "Floating of the transducer" in this manner also maximizes energy delivery to the fluid. The centerpiece and the two support structures are fastened together, with an airtight seal created around the fluid by the latex membranes that are glued around the transducer and reflector. The distance between the transducer and reflector and the planar position of one relative to the other can be fine-tuned by adjusting the setscrews attached to the positioning plates. This versatility is necessary since the lineup of cells at the nodal position at the fundamental frequency is strongly influenced by reflector and transducer positions.

The piezoelectric transducer (PZT, Navy Type I, EDO Electro Ceramics, and Model EC-64) had dimensions $10.3 \times 37.7 \times 74.6 \mathrm{~mm}$. The chamber dimensions were determined according to the general guidelines developed by Groschl (1998) and modified by Rusinko (2000). The operating frequency $f_{0}$ was selected to be the fundamental frequency of the piezoelectric layer, $210 \mathrm{kHz}$, in order to maximize the chamber width. The fluid layer thickness was $3.55 \mathrm{~mm}$, equal to $1 / 2$ wavelength; the stainless steel reflector was machined to a thickness of $4.1 \mathrm{~mm}$, with the same length and width as the transducer.

Assuming fully developed parabolic flow in each portion of the chamber, the ratio of flow rates of the carrier and feed streams $\left(Q_{1}\right.$ and $Q_{2}$, respectively) can be derived as a function of $S$, the inlet splitter position and $b$, the halfthickness of the chamber, resulting in:

$$
\frac{Q_{1}}{Q_{2}}=\frac{(3 b-S) S^{2}-4 b^{3}}{S^{2}(S-3 b)}
$$

In practice, $Q_{1}$ and $Q_{2}$ are constrained by the pump capacities. Using $Q_{1}=8 \mathrm{ml} / \mathrm{min}$ and $Q_{2}=0.6 \mathrm{ml} / \mathrm{min}$, and with $b$ determined from the chamber width (described above), $S$ was set to $0.56 \mathrm{~mm}$. Since the actual flows within the chamber are not fully developed, the pumping rates were then fine-tuned until visual observations showed that the feed suspension did not flow mix into the carrier stream when no acoustic field was activated. Spectrophotometer measurements were used to corroborate visual observations. The actual flow rates used in the fractionation experiments were 8.0 and $0.4 \mathrm{ml} / \mathrm{min}$ for $Q_{1}$ and $Q_{2}$, respectively.

\section{Cell Suspension Preparation}

The hybridoma cells (cell line SF1-1.1.1, Anti H-2Kd, ATCC\#HB-159, American Type Culture Collection, Rockville, MD) were cultured in RPMI media (Sigma-Aldrich, St. Louis, MO) with 5\% fetal bovine serum (SigmaAldrich) at a temperature of $37^{\circ} \mathrm{C}$ and maintained in a humidified incubator and 5\% $\mathrm{CO}_{2}$ atmosphere in T-25 culture flasks. The media was replaced once every 2 days. Cell viability immediately prior to experiments ranged between 95-99\%, as measured using a hemocytometer and Trypan blue stain. The concentration of the hybridoma cell suspension used in experiments was $\sim 1.2 \times 10^{6}$ cells $/ \mathrm{mL}$.

Lactobacillus rhamnosus ATCC (7469) strain in \#416 was cultured anaerobically in lactobacilli MRS broth (Difco, Detroit MI, 288130) at $37^{\circ} \mathrm{C}$. The concentration of the lactobacillus cell suspension used in experiments was $\sim 4.8 \times 10^{7}$ cells $/ \mathrm{mL}$.

\section{Experimental Procedure}

A schematic of the general system setup is shown in Figure 3. A BioRad (Hercules, CA) Econo-column 56BR5193 peristaltic pump was used to deliver the feed suspension to the chamber. A Masterflex 7250-10 peristaltic pump was used to deliver the carrier fluid to the chamber and a Masterflex 7250-10 peristaltic pump pulled the suspension from the right outlet of the chamber. The use of the third pump was necessary to control the flow and minimize crossover between the feed and carrier fluid streams. Pulse dampeners on both the feed and the carrier streams smoothed the flow generated by the action of the peristaltic pumps. A magnetic stirrer in the feed stream kept the cell suspension uniformly mixed. A Fluka 6011A synthesized signal generator (Fluka, Everett, WA) was used to generate the signal, which was amplified by an ENI 240L power amplifier (ENI, Andover, MA). 


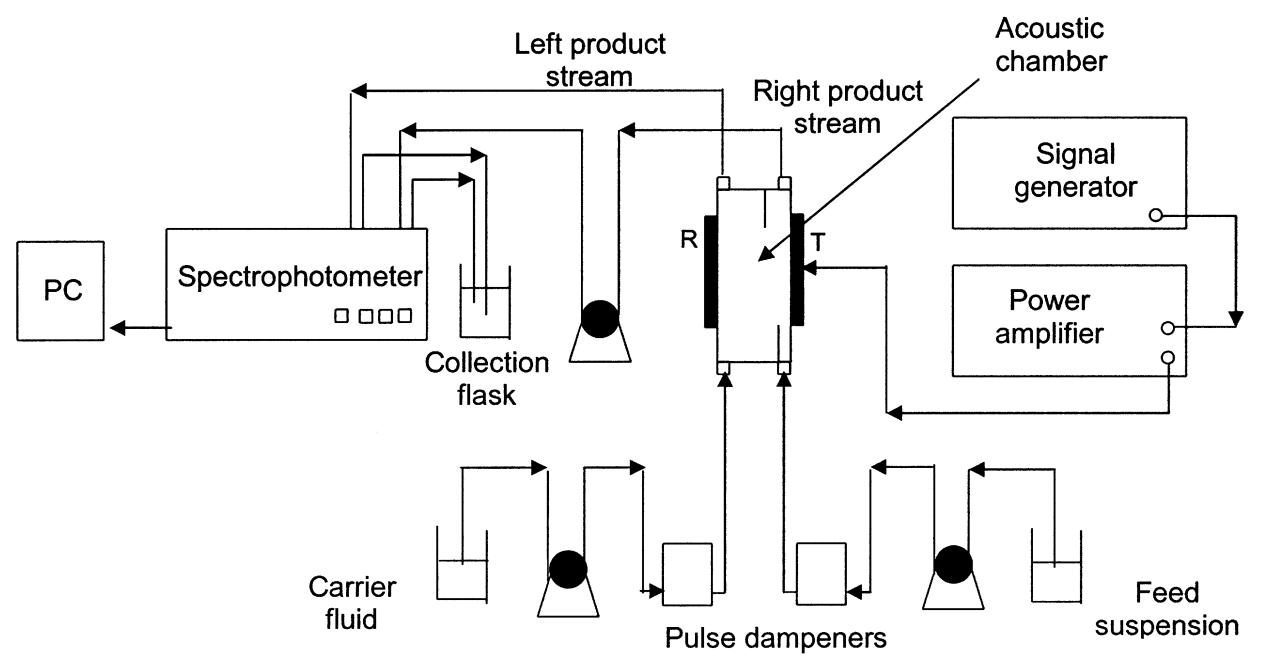

Figure 3. Schematic of the experimental configuration.

In experiments using single cell populations, each of the outlet streams was connected to a quartz flow-through semi-micro cuvette (Hellma Cells, Plainview, NY) and a Milton Roy Spectronic 1001 Plus spectrophotometer (Rochester, NY) was used to measure absorbance at $300 \mathrm{~nm}$. The absorbance was found to be linear with respect to cell concentration for both cell types. The spectrophotometer was interfaced to a PC that continuously recorded absorbance. Samples of the pure populations were analyzed for size distribution in the laser diffraction based particle size analyzer (Malvern Instruments, Southborough, MA).

The feed stream in the fractionation experiments consisted of hybridoma cell suspension mixed with lactobacillus cell suspension in a 4:1 suspension volume ratio. The experimental system was allowed to stabilize for $30 \mathrm{~min}$ at each operating condition before collecting the samples. A continuous sequence of samples from the outlet streams (each one representing $5 \mathrm{~min}$ of flow) was obtained and each sample was analyzed in the laser diffraction particle size analyzer.

\section{RESULTS AND DISCUSSION}

Initial experiments to verify the operation of the separation chamber were performed with feed streams containing only one type of cell. The results of these experiments are shown in Table I. The fractions of cells leaving the chamber that exit in either the left or right product streams (defined as the recovery \%) are calculated from the exit concentration measurements and flow rates. For no acoustic field (signal generator voltage $=0$ ), the recovery in the right product stream of lactobacilli and hybridomas are $95.1 \pm 0.3 \%$ and $91.3 \pm 0.3 \%$, respectively, indicating that there is minimal crossflow between the feed and carrier streams. When the signal generator is set to $10 \mathrm{mVpp}$, the recovery of lactobacilli in the right product stream remains high, at $95.0 \pm$ $1.5 \%$, but drops to $90.5 \pm 2.1 \%$ at $25 \mathrm{mVpp}$. At higher energy levels (50 mVpp signal), more lactobacilli reach the nodal plane, with the recovery dropping to $88.6 \%$. For a signal generator voltage of $10 \mathrm{mVpp}$, the recovery of the much-larger hybridomas in the left product stream is $82.7 \pm$ $2.8 \%$, and increases to $91.2 \pm 1.0 \%$ at $25 \mathrm{mVpp}$. These results demonstrate the feasibility of using this chamber as a separation device at signal voltages of $10-25 \mathrm{mVpp}$, since the fraction of cells reaching the nodal plane was significantly lower for lactobacilli compared to hybridomas at both of these conditions. The results obtained with no acoustic field provide a measure of quality of the hydrodynamic conditions within our experimental chamber. The slight $(5-10 \%)$ crossover can be attributed to imperfect con-

Table I. Recovery of lactobacillus and hybridomas from individual cell suspensions in the left and right product streams (mean $\pm 95 \%$ confidence limits); $\mathrm{n}=$ the number of experiments.

\begin{tabular}{|c|c|c|c|c|c|c|c|}
\hline \multirow{2}{*}{$\begin{array}{l}\text { Acoustic energy density Eac } \\
\qquad \mathrm{kg} / \mathrm{mm} \cdot \mathrm{s}^{2}\end{array}$} & \multirow{2}{*}{$\begin{array}{l}\text { Signal generator voltage } \\
\mathrm{mVpp}\end{array}$} & \multicolumn{3}{|c|}{ Relative amounts of lactobacilli (\%) } & \multicolumn{3}{|c|}{ Relative amounts of hybridomas } \\
\hline & & & Left & Right & & Left & Right \\
\hline 0 & 0 & $\mathrm{n}=3$ & $4.9 \pm 0.0$ & $95.1 \pm 0.3$ & $\mathrm{n}=3$ & $8.7 \pm .1$ & $91.3 \pm 0.3$ \\
\hline 0.0030 & 10 & $\mathrm{n}=3$ & $5.0 \pm 0.0$ & $95.0 \pm 1.5$ & $\mathrm{n}=3$ & $82.7 \pm 2.8$ & $17.3 \pm 0.1$ \\
\hline 0.0056 & 25 & $\mathrm{n}=3$ & $9.5 \pm 0.0$ & $90.5 \pm 2.1$ & $\mathrm{n}=3$ & $91.2 \pm 1.0$ & $8.8 \pm 0.5$ \\
\hline 0.0125 & 50 & $\mathrm{n}=1$ & 11.4 & 88.6 & $\mathrm{n}=3$ & $88.0 \pm .4$ & $12.0 \pm .4$ \\
\hline N/A & 100 & - & - & & $\mathrm{n}=1$ & 57.1 & 42.9 \\
\hline
\end{tabular}


trol by the metering pumps, blunt edges of the flow splitters, or slight misalignments of the chamber. Nevertheless, the experimental apparatus proved to be more than adequate to demonstrate the desired fractionation.

For the signal generator operated in the range 0$25 \mathrm{mVpp}$, a total of $98-100 \%$ of the cells fed to the chamber were observed in the outlet streams as calculated from a mass balance around the chamber (data not shown). The total amount of cells observed in the effluent streams dropped to $86 \%$ and $81 \%$ for lactobacilli and hybridomas, respectively, at a signal voltage of $50 \mathrm{mVpp}$, and these values dropped further to $61 \%$ for hybridomas for a $100 \mathrm{mVpp}$ signal voltage. Cell clumping was observed within the acoustic fields generated with the signal generator set at 50 and $100 \mathrm{mVpp}$. Such clumps settled downward against the flow and accumulated in the chamber.

The recoveries in the left output stream from Table I are plotted in Figure 4 against the corresponding $D$ values using $5.23 \mu \mathrm{m}$ and $0.61 \mu \mathrm{m}$ as the cell diameters for the hybridomas and lactobacillus, respectively. These values were based on the area-mean diameter $\left(P_{A M}\right)$ calculated from the size distributions of the pure populations (Fig. 5) using the equation:

$$
P_{A M}{ }^{2}=\frac{\int_{P 1}^{P 2} P^{2} n(P) d P}{\int_{P 1}^{P 2} n(P) d P}
$$

where $P$ is the diameter and $n(P)$ is the number of particles at that diameter. Because $D$ depends on the cell radius squared, the hybridoma data lie in the $D$ range two orders of magnitude greater than that of lactobacillus. Yields at $D=0$ (no sound) are actually plotted at $D=0.001$ for convenience on the semi-log plot. Figure 4 also contains

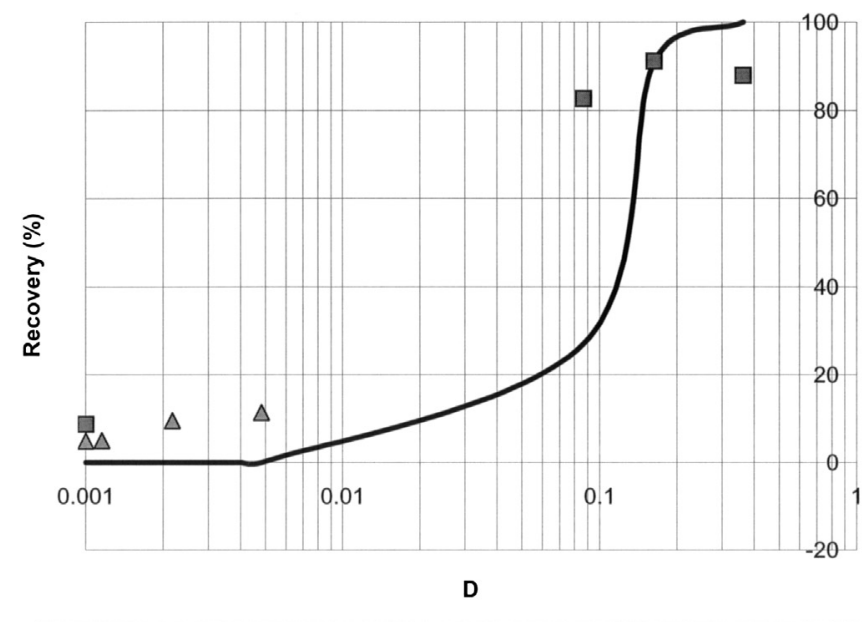

Figure 4. Recovery of cells in left product stream, from data in Table I, plotted against dimensionless parameter $D$. Data are from experiments with pure cell populations. The theoretical result is calculated from the model described in the text.

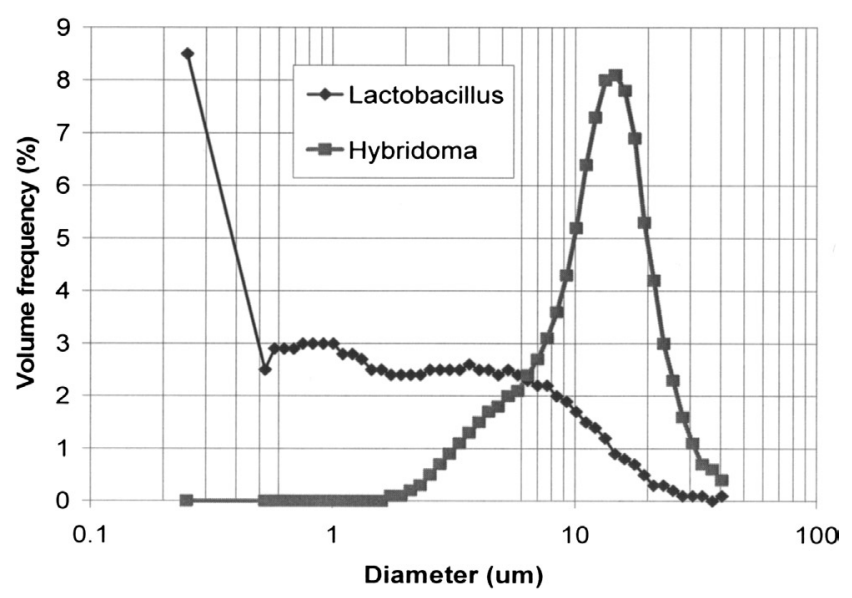

Figure 5. Volume frequency distribution of the pure cell populations (hybridoma and lactobacillus).

the theoretical recovery curve calculated using Eqs. 6 and 8 and the procedure discussed previously. In general, the trajectory model does a very good job of predicting the recovery of the two cell lines. The small number of lactobacilli that exited in the left stream may result from some mixing between the streams. Furthermore, the model assumes that the cells are perfect spheres, whereas the lactobacilli are more rod-shaped and form chains. The higher-than-expected recovery of hybridomas in the left stream at $D=0.09$ (signal voltage $=10 \mathrm{mVpp}$ ) may result from dispersion effects which are not considered in the model. Discrepancies may also be due to the model assumption that the cells are uniformly distributed across the inlet position and that the cells within each population are of uniform size.

Figures 5 and 6 show the size distributions of the pure population samples and the cell mixture used as the feed in the separation experiments, respectively. Note that because this is a volume distribution, the distribution appears to be skewed more towards the larger cells. The particle size analyzer output is in \%volume frequency, and in order to avoid the introduction of additional assumptions and potential errors (such as assuming perfectly spherical cells), the

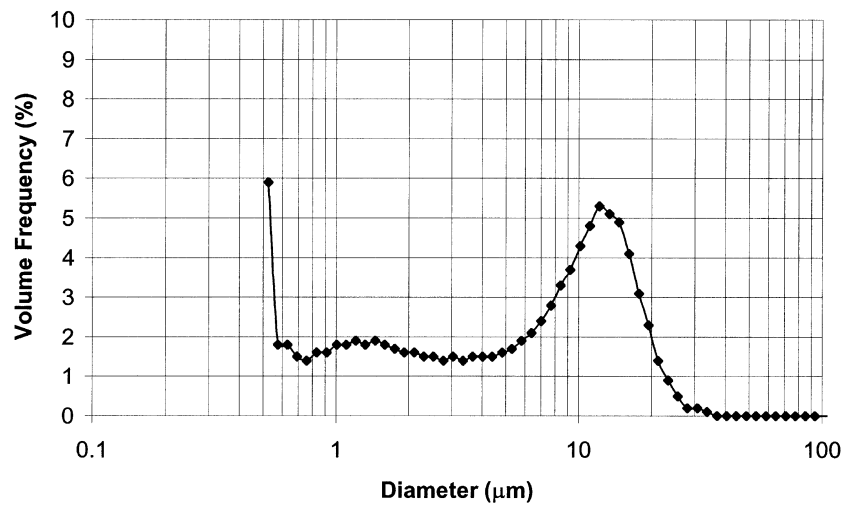

Figure 6. Volume frequency distribution of hybridoma/lactobacillus mixture used as the feed stream in separation experiments. 


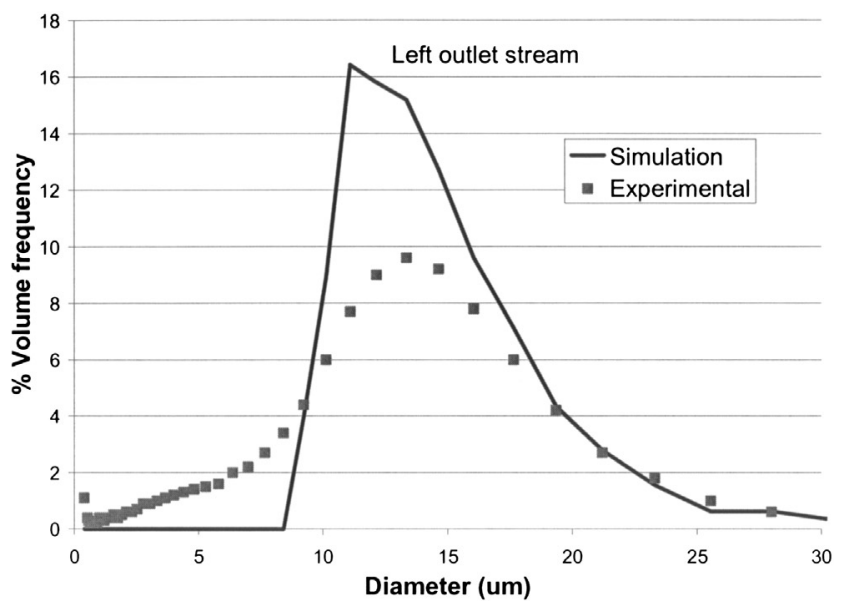

Figure 7. Comparison of experimental to model results of volume frequency distribution in left product stream for a $25 \mathrm{mVpp}$ signal.

results are reported directly in the same units. The size distributions of the two outlet streams when $25 \mathrm{mVpp}$ signal was applied are shown in Figures 7 and 8. From a comparison of these two figures we can clearly see that the left product stream contained mostly the larger hybridomas and the right stream contained mostly the smaller cells, including the lactobacillus.

The trajectory model can be used to provide a prediction of the population distributions of the two exit streams based on an assumed composition of the feed mixture. For this purpose, we assumed the feed stream consisted of 100 cells with diameters varying from $0.40-30.7 \mu \mathrm{m}$, with the diameter distribution matching the particle size distribution (Fig. 6) of the feed suspension used in the actual experiments. The cells were assumed to be uniformly distributed across the $\mathrm{x}$-direction at the chamber inlet. The exit position for each cell was determined for each cell size and inlet position. The number size distribution expected in each product stream was calculated and subsequently converted to a volume distribution.

The theoretical results are shown along with the experimental results in Figures 7 and 8. Experimental and

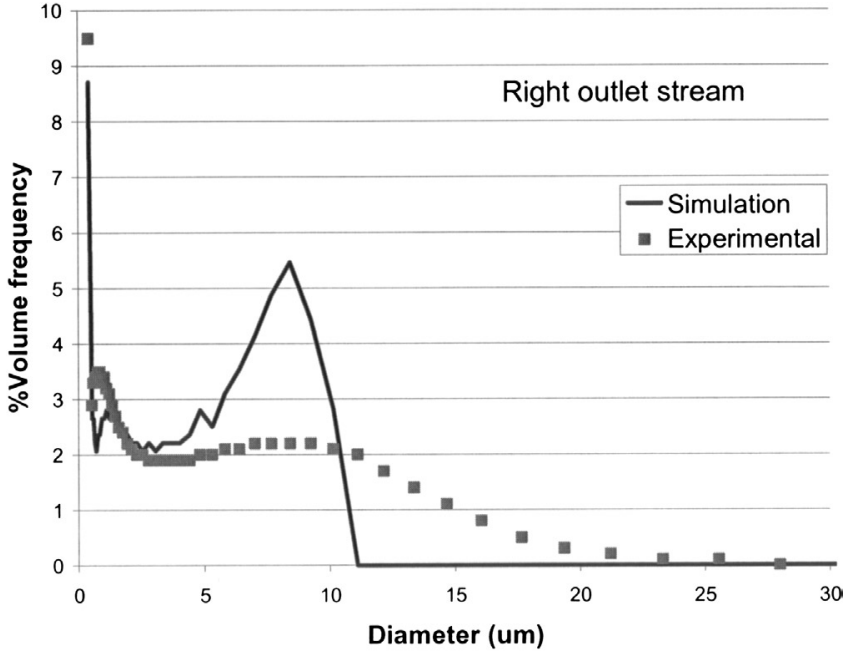

Figure 8. Comparison of experimental to model results of volume frequency distribution in right product stream for a $25 \mathrm{mVpp}$ signal.

theoretical results are very similar for the left product stream (Fig. 7) except that the peak is sharper in the theoretical result, which is to be expected since the model does not include any nonidealities in fluid flow. In the right product stream (Fig. 8), some of the larger cells remained on the right side of the splitter, whereas in the simulation, none would be expected. Some of the discrepancy may arise from the finite thickness of the exit splitter, which may prevent some of the larger, faster-responding cells from exiting in the left product stream as theoretically predicted, while in the model, the splitter is assumed to occupy zero thickness. The discrepancy could also be due to cell clumping, dispersion, the flow not fully developed or pulsation in the flow, or uneven distribution of cells in the inlet.

In order to quantify the fractionation achieved in the experiments, we define the selectivity to be the ratio of hybridomas to lactobacilli in an exit stream, either on a volume basis or cell number basis. The normalized selectivity is the selectivity of the output stream normalized to that of the feed stream. The choice of a cutoff diameter, to distinguish the hybridomas from the lactobacillus, was

Table II. Ratio of hybridoma/lactobacillus of each product stream, normalized to that of the feed stream (normalized selectivity), on cell volume and cell number basis, using three different cutoff sizes to distinguish between the two cell types; results are at $25 \mathrm{mVpp}$.

\begin{tabular}{|c|c|c|c|c|c|}
\hline \multirow{2}{*}{$\begin{array}{l}\text { Calculation } \\
\text { basis }\end{array}$} & \multirow{2}{*}{$\begin{array}{c}\text { Cutoff } \\
\text { diameter }(\mu \mathrm{m})\end{array}$} & \multicolumn{2}{|c|}{ Normalized selectivity } & \multicolumn{2}{|c|}{$\begin{array}{c}\text { Expected normalized } \\
\text { selectivity based on } \\
\text { Table I results }\end{array}$} \\
\hline & & Left & Right & Left & Right \\
\hline \multirow[t]{3}{*}{ Volume } & 1.0 & $6.6 \pm 1.5$ & $0.44 \pm 0.02$ & \multirow[t]{3}{*}{9.6} & \multirow[t]{3}{*}{0.097} \\
\hline & 2.0 & $6.9 \pm 1.8$ & $0.39 \pm 0.01$ & & \\
\hline & 5.0 & $4.8 \pm 0.9$ & $0.33 \pm 0.02$ & & \\
\hline \multirow[t]{3}{*}{ Cell number } & 1.0 & $1.3 \pm 0.3$ & $0.96 \pm 0.03$ & \multirow[t]{3}{*}{9.6} & \multirow[t]{3}{*}{0.097} \\
\hline & 2.0 & $2.8 \pm 0.3$ & $0.72 \pm 0.02$ & & \\
\hline & 5.0 & $5.6 \pm 0.9$ & $0.50 \pm 0.11$ & & \\
\hline
\end{tabular}


not obvious because of the relatively flat distribution of the lactobacillus population (Fig. 5). At $2.0 \mu \mathrm{m}$ cutoff, $49.8 \%$ of the lactobacillus in the mixture will be included in the lactobacillus population, while $99.8 \%$ of the hybridomas are included in the hybridoma population. At a larger cutoff value, the lactobacillus population will include a greater proportion of cells, while the hybridoma population will include a smaller proportion of cells. Using the $2.0 \mu \mathrm{m}$ cutoff and the data in Figure 6, the hybridoma/lactobacillus ratio (based on cell volume) of the feed stream is calculated to be $2.18 \mathrm{vol} / \mathrm{vol}$.

At $10 \mathrm{mVpp}$ and using the $2.0 \mu \mathrm{m}$ cutoff, the normalized selectivities in the left and right product streams were $3.20 \pm$ $0.02(\mathrm{vol} / \mathrm{vol})$ in the left product stream and $0.496 \pm$ $0.001(\mathrm{vol} / \mathrm{vol})$ in the right product stream $(\mathrm{n}=2)$, indicating that some degree of fractionation was achieved. At the higher energy level of $25 \mathrm{mVpp}$, the normalized selectivities were $6.9 \pm 1.8$ and $0.39 \pm 0.01(\mathrm{vol} / \mathrm{vol})(\mathrm{n}=$ 4 ) in the left and right product streams, respectively. The large difference between these two ratios indicates that a high degree of fractionation of the two cell populations was achieved and that the separation improved at the higher energy level. At smaller $(1 \mu \mathrm{m})$ or larger $(5 \mu \mathrm{m})$ cutoff values, the actual selectivities change, but there remains a 15-18-fold increase in normalized selectivity between the right and left output streams (Table II). When calculated on a cell number/number basis, the difference in normalized selectives between left and right are much more sensitive to the selection of the cutoff diameter and the separation does not appear as dramatic as that calculated on the volume basis (Table II). Nevertheless, fractionation of cells into the two populations is still clear.

The cell recoveries from the individual population experiments (Table I) were used to calculate the expected normalized selectivities in the mixed cell suspension experiments. The results, shown in Table II, indicate that the achieved fractionation was certainly less than that expected, but they were in the proper range. The differences could be accounted for by interaction of the cells with the acoustic field, imperfections in the hydrodynamic conditions, or possibly by inadequacies in the measurement techniques and assumptions in assigning cells to either the hybridoma or lactobacillus populations.

\section{CONCLUSIONS}

A technique for the fractionation of a mixture of two different populations of cells using a combination of laminar flow fields and resonant acoustic fields has been identified. A theoretical model of the process was first developed to calculate the cell trajectories of different sized cells and the expected size distributions in each of the two product streams given a mixed cell population in the feed. Compared to experimental data, the model provided good estimates of the final position of cells from pure cell suspensions, signifying that it would be useful as a tool for identifying operating conditions necessary to fractionate mixtures of cell populations. The model predicted a nearly complete separation of the hybridoma and lactobacillus cell populations at signal generator voltage of $25 \mathrm{mVpp}$ (corresponding to acoustic energy densities of $0.0056 \mathrm{~kg} /$ $\mathrm{mm} \cdot \mathrm{sec}^{2}$ ). Experiments conducted using these conditions resulted in normalized hybridoma/lactobacillus ratios in the left and right product stream of $6.9 \pm 1.8$ and $0.39 \pm$ $0.01(\mathrm{vol} / \mathrm{vol})$, respectively, indicating that the cell types were separated to a significant extent.

The advantage of the approach presented here is that cell populations can be separated based on purely physical properties (i.e., diameter, compressibility, and density) in an efficient, scaleable process, without the need for immunolabeling. Numerous works in the literature have shown that short-term exposure of cells to the levels of sound intensity used here do not have detrimental effects on cell viability (Gaida et al., 1996; Ryll et al., 2000; Wang et al., 2003). Improvement in the fractionation can be expected by optimization of the operating parameters and chamber geometry. Higher-resolution fractionations could be achieved with several acoustic-flow chambers combined in series so as to develop a multistage process. Such a multistage device should be able to then separate populations of cells that are closer in diameter to each other than the hybridoma/ lactobacillus mixture that was tested here in these proof-ofconcept experiments.

Scale-up of this concept to larger flow rates can be achieved in a number of ways. These include increasing the width $(b)$ or the depth (z-direction dimension) of the chamber or by operating multiple chambers in parallel. By stacking the chambers in the $z$-direction, multiple devices could be driven by a single transducer. A control system to maintain the system at resonance would be needed to ensure long-term stable operation of the device. The theoretical curve in Figure 4 provides a means to estimate whether separation can occur for a given set of cells and to identify the window of operating conditions to obtain this separation.

\section{References}

Davis RH, Lee C, Kompala DS. 1991. Cell separations using differential sedimentation in inclined settlers. ACS Symposium Series 464: $113-127$.

Gaida T, Dolbhoff-Dier O, Strutzenberger K, Burger W, Groschl M, Handl B, Benes E. 1996. Selective retention of viable cells in ultrasonic resonance field devices. Biotechnol Prog 12:73-76.

Giddings JC. 1988. Continuous separation in split-flow (SPLITT) cells: potential applications to biological materials. Separ Sci Tech 23: 931-943.

Giddings JC. 1992. Optimization of transport-driven continuous SPLITT fractionation. Sep Sci Tech 27:1489-1504.

Giddings JC, Barman BN, Liu M. 1991. Separation of cells by field flow fractionation. In: Kompala DS, Todd P, editors. Cell separation science and technology. American Chemical Society. p 128-144.

Groschl M. 1998. Ultrasonic separation of suspended particles. II. Design and operation of separation devices. Acustica 84:632-642.

Grossner MT, Feke DL, Belovich JM. 2004. Single collector experiments and modeling of acoustically aided mesh filtration. AIChE J (in press). 
Hartig R, Becker HD, Liebich HG. 1996. Enrichment of gastric endocrine cells by continuous immuno magnetic separation. Regul Pept 64:63.

Hawkes JJ, Barrow D, Coakley WT. 1998a. Microparticle manipulation in millimeter scale ultrasonic standing wave chambers. Ultrasonics 36 : 925-931.

Hawkes JJ, Barrow D, Coakley WT, Cefai J. 1998b. A laminar flow expansion chamber facilitating downstream manipulation of particles concentrated using an ultrasonic standing wave. Ultrasonics 36: 901-903.

Johnson DA, Feke DL. 1995. Methodology for fractionating suspended particles using ultrasonic standing wave and divided flow fields. Separ Technol 5:251-258.

King LV. 1934. On the acoustic radiation pressures on spheres. Proc R Soc Lond 147A:212-240.

Mandralis ZI, Feke DL. 1993. Continuous suspension fractionation using acoustic and divided flow fields. Chem Eng Sci 48:3897-3905.

Mandralis ZI, Adler RJ, Feke DL. 1990. Transient response of fine particle suspensions to mild planar ultrasonic fields. Fluid Particle Separ J 3:115-121.

Pangu GD, Feke DL. 2004. Acoustically aided separation of oil droplets from aqueous emulsions. Chem Eng Sci 59:3183-3193.

Rusinko D. 2000. Design and optimization of an ultrasonic standing wave chamber. MS Thesis, Case Western Reserve University, Cleveland, $\mathrm{OH}$.
Ryll T, Dutina G, Reyes A, Gunson J, Krummen L, Etcheverry T. 2000. Performance of small-scale $\mathrm{CHO}$ perfusion cultures using an acoustic cell filtration device for cell retention: characterization of separation efficiency and impact of perfusion on product quality. Biotechnol Bioeng 69:440-449.

Sharma R, Edwards R, Beckett R. 1998. Analysis of bacteria in aquatic environments using sedimentation field-flow fractionation. II. Physical characterization of cells. Water Res 32:1508-1514.

Simon H, Gregor Z, Qing Y, Paul VR. 1998. Fluorescence activated cell sorting: a reliable method in tissue engineering of a bioprosthetic heart valve. Ann Thorac Surg 66:1653-1657.

Svoboda J. 2000. Separation of red blood cells by magnetic means. J Magn Magn Mater 220:103-105.

Toubal M, Asmani M, Radziszewski E, Nongaillard B. 1999. Acoustic measurement of compressibility and thermal expansion coefficient of erythrocytes. Phys Med Biol 44:1277-1287.

Wang Z, Grabenstetter P, Feke D, Belovich J. 2003. Retention and viability characteristics of mammalian cells in an acoustically driven porous mesh. Biotechnol Prog 20:384-387.

Ximena R, Chris H, Pierre K, Martine D. 1997. Purification and characterization of bovine dendritic cells from peripheral blood. Vet Immunol Imunopathol 60:77-88.

Yosioka K, Kawasima Y. 1955. Acoustic radiation pressure on a compressible sphere. Acustica 5:167-173. 